\title{
Simulation and Experimental Validation of the Hot Embossing Process of Poly(lactic-co-glycolic acid) Microstructures
}

\author{
Xiaopeng Wang, Wei Li, and Tianning Chen \\ School of Mechanical Engineering, State Key Laboratory for Strength and Vibration of Mechanical Structures, \\ Xian Jiaotong University, Xi'an 710049, China \\ Correspondence should be addressed to Xiaopeng Wang; xpwang@mail.xjtu.edu.cn
}

Received 18 August 2014; Accepted 26 December 2014

Academic Editor: Long Yu

Copyright (C) 2015 Xiaopeng Wang et al. This is an open access article distributed under the Creative Commons Attribution License, which permits unrestricted use, distribution, and reproduction in any medium, provided the original work is properly cited.

The microstructures were fabricated by hot embossing method using biodegradable material PLGA poly(lactic-co-glycolic acid), to serve as the drug carriers in the drug delivery system. The embossing process was studied in a combination of simulations and experiments. Firstly, the viscoelastic model of PLGA was built after testing the material properties. Secondly, the hot embossing process was simulated by ABAQUS finite element software. The deformation rules of PLGA during hot embossing were then achieved. The pressures inside the PLGA materials were different at various places during hot embossing, which lead to the differences of the filling speeds. As a result, the inner structures were easier to get formed than the outer structures. And the fluidity of PGLA would increase with the raising temperature, which however caused serious material overflow. Finally the hot embossing experiments were presented to verify the simulation results. Agreed with the filling rules of the simulation, enough duration was necessary to let the outer corners of the microstructures be formed completely. Moreover the trapped air in the grooves was compressed into small bubbles at the corners. It was also found that the material overflow could be prevented in the use of nonisothermal hot embossing method.

\section{Introduction}

Hot embossing is one of the most important techniques to fabricate the polymer microstructures, especially suitable for large-area thin components and structures with extreme aspect ratios. Hot embossing involves heating the polymer material, embossing the polymer with the patterned mold, cooling the system with the embossing load being maintained, and finally separating the finished part from the mold. Considering its advances of low cost and simple operation, it has been applied vastly in the areas like MEMS (Microelectromechanical Systems) and biomedical engineering [1].

Researches on the polymer hot embossing process were focused on the materials of PMMA (polymethyl methacrylate) and PC (polycarbonate) using simulation and experimental methods. Worgull et al. established the viscoelastic model of the PMMA based on the materials traction tests and WLF equation to perform the embossing simulation [2]. The viscous fluid model [3] and the plastic model [4] were also applied to simulate the material properties during hot embossing. The material would fill the grooves in the forms of single peak and dual peaks according to the heightwidth ratio of the microstructures. Rowland and King did the researches on the relations between the peak height and the embossing temperature [5]. The multichannel or multigroove structures were often simplified as symmetrical single-channel or single-groove model in most of the simulations, which neglected the effect of pressure differences. He et al. found that the nonuniform pressure distribution can result in the pattern height difference when simulating the embossing process [6].

Experimental studies of hot embossing process were focused on the influences of different embossing parameters, including pressure, temperature, duration, and initial thickness. Researches by Lan et al. showed that the embossing temperature was more important for the qualities of microstructures than the others [7]. Heyderman et al. established an experienced equation to calculate the duration 
needed to fill the structures [8]. Hirai et al. pointed out that inappropriate height-width ratio and over small initial thickness would impede the embossing process [9].

One of the main defects of embossed microstructures is the existence of bubbles, which are caused by the trapped air in the pattern grooves and cavities. The trapped air can also impede the pattern replication during hot embossing. Taylor et al. presented a fast simulation technique that captures the impact of trapped air in the microembossing process [10]. Morihara et al. conducted the research on the influence of material viscosity on the bubbles formation [11]. There are generally two ways to avoid the bubble defects: (1) installing the vacuum chamber; the vacuum environment can improve the uniformity of microstructures qualities, which however adds to a much higher cost and complexity [12]; (2) using less stiff mold such as PDMS (polydimethylsiloxane) instead of metal mold to squeeze the air out $[13,14]$.

Most of the scholars did researches on isothermal hot embossing, in which the plate (or substrate) and the patterned mold would be heated to the same temperature during embossing process. Isothermal method is also the most popular process for the hot embossing of polymers. In contrast, the nonisothermal embossing involves heating the mold and the plate to different temperatures, which has not been given sufficient attention and has been less discussed. Juang et al. found that the replication accuracy of nonisothermal embossing is not strongly dependent on the processing conditions compared with the isothermal way; as long as the pattern transfer is completed, the replication accuracy is excellent since a high temperature can be achieved locally [15]. Yao et al. studied the influence of the cavity size on the heat diffusion process and the filling process during nonisothermal embossing [16]. Hug et al. fabricated the optical lenses by nonisothermal embossing of inorganic glasses and investigated the process parameters [17].

Recently, many novel polymeric microdevices used to deliver drugs have been invented with the help of hot embossing technology [18-20]. Our team has also proposed a novel implantable drug delivery system, as shown in Figure 1. The biodegradable material PLGA (poly(lactic-co-glycolic acid)) is fabricated into the multicavities microstructures by hot embossing method, to serve as the drug carriers of the drug delivery system. Then the embossed microstructures are sealed by a PLGA thin film with microholes and implanted into human body to deliver drugs according to predefined rules. Besides, the relationships between the drug delivery rules and the dimensions of the microstructures had also been studied by our team earlier before [21, 22]. The novel drug delivery system can relieve the suffering of the patients and improve the treatment effect dramatically because of its many commendable benefits such as targeted releasing, rate controlling, large capacity, and long period.

We used the PDMS as the material of embossing mold in the early time, but the PDMS mold deformed seriously while applying large load and its service life was also limited. Then the metal mold of copper material was used to replace the PDMS mold to avoid its disadvantages. But some new problems, such as bubbles defects, material overflow, and uncertainty of process parameters, were introduced during

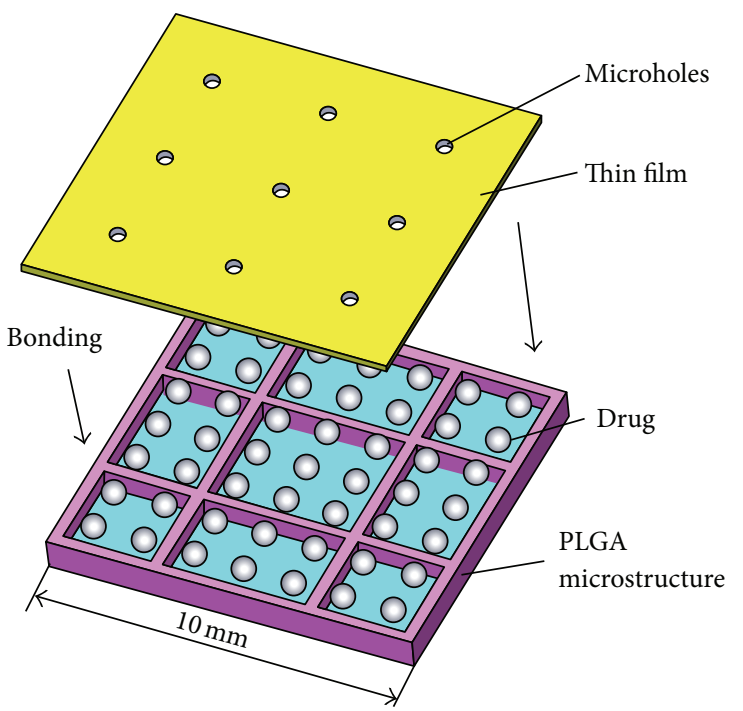

FIgURe 1: PLGA drug delivery system.

the fabrication. Since the embossing process based on metal mold was not mature yet, it is very necessary to investigate these problems to get the stable, complete, and qualified microstructures.

\section{Material Properties}

2.1. Viscoelastic Model. PLGA has the glass transition temperature of about $45^{\circ} \mathrm{C}$ and the embossing temperature of 60 to $90^{\circ} \mathrm{C}$. The mechanical behavior of PLGA above the glass transition temperature is not only temperature-dependent but also time-dependent, presenting the viscoelastic property. In this situation, the generalized Maxwell model is used to describe the molding behavior of viscoelastic materials in hot embossing, in which springs and damps are used to simulate the linear elastic response and the linear viscous response, respectively, as shown in Figure 2. The time dependent relaxation modulus, $E(t)$, can be expressed as follows [23]:

$$
\begin{gathered}
E(t)=E_{\infty}+\sum_{i=1}^{n} E_{i} \exp \left(-\frac{t}{\tau_{i}}\right), \\
\tau_{i}=\frac{\eta_{i}}{E_{i}},
\end{gathered}
$$

where $E_{\infty}$ is the equilibrium value of the relaxation modulus of $E(t)$ after the time goes to infinity, $n$ is the number of dampspring units, $E_{i}$ is the modulus of the spring, $\eta_{i}$ is the viscosity of the damp, and $\tau_{i}$ is the relaxation time. Until now, there are no research data about PLGA relaxation modulus, so the following experiments are conducted to confirm the values of the Maxwell model.

2.2. Stress Relaxation Experiments. The PLGA material (monomer ratio PLA/PGA is 50/50) used in the stress relaxation experiments was purchased from LACTEL USA. PLGA test samples of $30 \times 5 \times 1.5 \mathrm{~mm}$ were fabricated in 


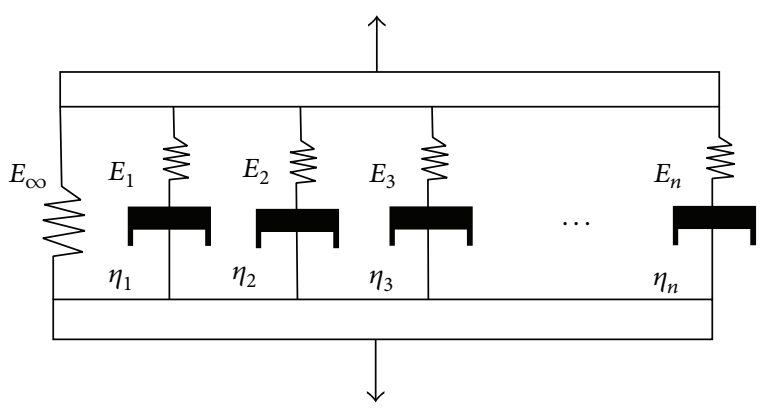

FIGURE 2: Generalized Maxwell model.

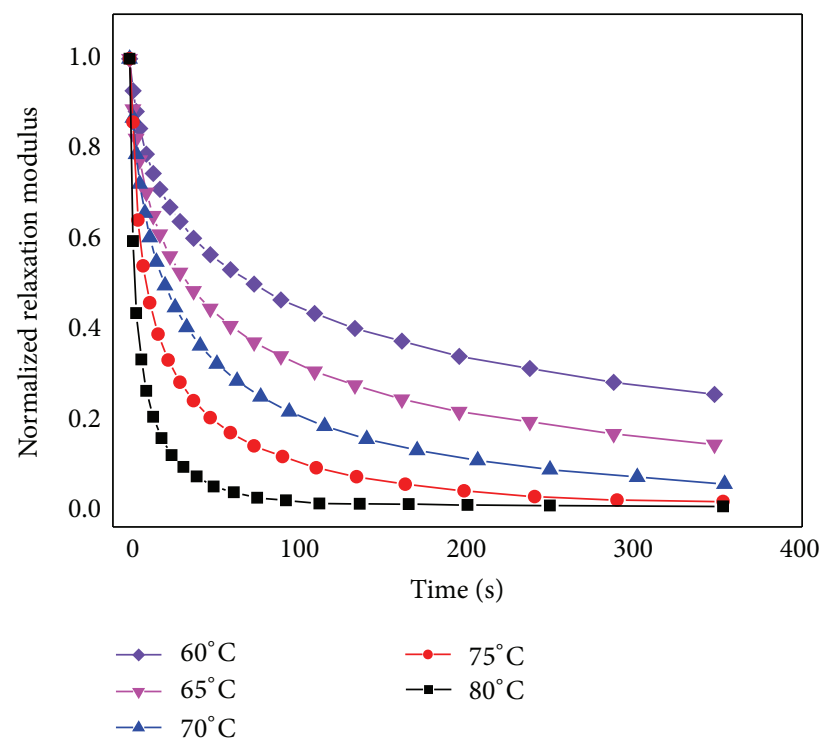

Figure 3: Normalized relaxation modulus curves of PLGA (50/50) with different temperatures.

advance. Dynamic mechanical analyzer (TA InstrumentsQ800) was used to measure the changes of the relaxation modulus. In experiments, the constant stretch strain was 1\% in length of the test sample, the relaxation time was $360 \mathrm{~s}$, the temperature range was $60-80^{\circ} \mathrm{C}$, and the temperature interval was $5^{\circ} \mathrm{C}$. The experimental results about the PLGA normalized relaxation modulus in different temperatures are shown in Figure 3, where the $X$ axis is the relaxation time and the $Y$ axis is the normalized relaxation modulus, by which a normalization process was performed by dividing the measured stress in function of time $E(t)$ of the initial stress $E_{0}$.

As shown in Figure 3, the relaxation modulus reduced significantly as the time elapsed at every tested temperature. The decrease rate of the relaxation modulus was rapid in the early stage and slowed down as the time went by. On the other hand, the decrease rate of the relaxation modulus increased as the tested temperature rose, and the modulus decreased rapidly to a lower value and then kept unchanged at $80^{\circ} \mathrm{C}$. This is because the higher temperature increased the energy of polymer molecular movement, and the expansion of polymer volume occurred with the increasing free space

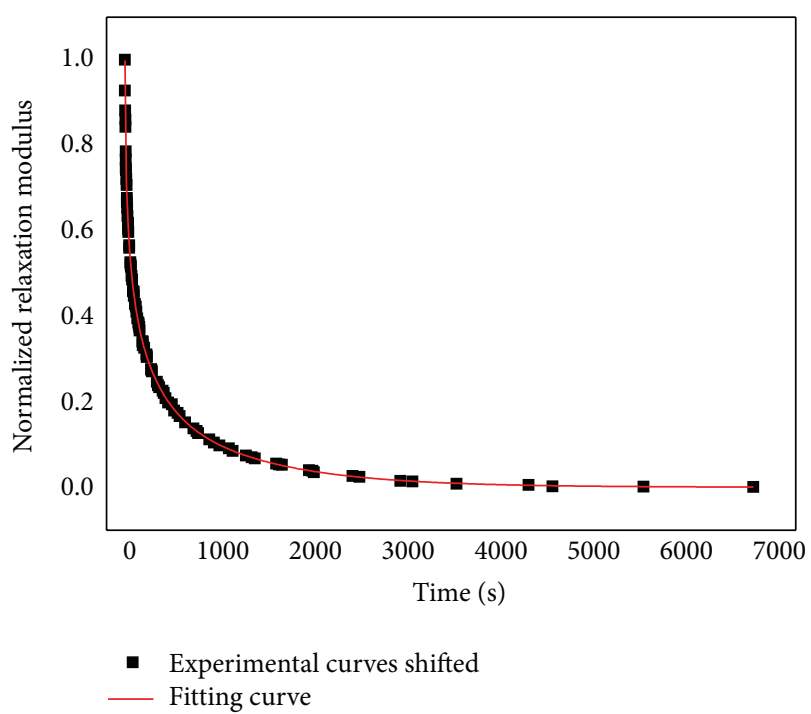

FIGURE 4: The master relaxation curve of PLGA and its fitting curve at $65^{\circ} \mathrm{C}$.

between molecules. Both effects speed up the process of relaxation and thus shorten the relaxation time.

2.3. The Master Curve of PLGA Stress Relaxation. According to the time-temperature equivalence characteristics of polymer materials, the relaxation behaviors are equivalent with the relatively short period of time at higher temperature and long period of time at lower temperature. Thus, if a reference temperature is selected, the relaxation curves at other temperatures can shift overlap. The overlapped relaxation curve is the master curve which shows the complete relaxation process for the polymer. For the amorphous polymer PLGA, the remove factor can be represented by the WLF equation [24]:

$$
\log _{10} a_{T}=\frac{-C_{1} \times\left(T-T_{0}\right)}{C_{2}+\left(T-T_{0}\right)}
$$

where $a_{T}$ is the remove factor, $T_{0}$ is the reference temperature and $T$ is measuring temperatures in relaxation experiment except the reference temperature, and $C_{1}$ and $C_{2}$ are the coefficients.

Accordingly, the master relaxation modulus curve of PLGA at $65^{\circ} \mathrm{C}$ can be obtained by moving the other relaxation curves at different temperatures in logarithmic coordinates based on Figure 3. And the removed curve is shown by square-symbol line in Figure 4. Meanwhile, the coefficients $C_{1}$ and $C_{2}$ can be got by substituting the remove factor to (2): $C_{1}$ is 8.2 and $C_{2}$ is 85.6.

So the WLF function of 50/50 PLGA at $65^{\circ} \mathrm{C}$ can be expressed as

$$
\log _{10} a_{T}=\frac{-8.2 \times\left(T-T_{0}\right)}{85.6+\left(T-T_{0}\right)}, \quad T_{0}=65^{\circ} \mathrm{C} .
$$

Then, the nonlinear fitting is conducted to the master curve of relaxation modulus, the number of Maxwell units, $n$, is 5 , and the fitting curve is shown by smooth-line 
TABLE 1: The parameters of generalized Maxwell model.

\begin{tabular}{lccc}
\hline Parameters & Fitting value & Parameters & Fitting value \\
\hline$\alpha_{\infty}$ & 0.00017 & - & - \\
$\alpha_{1}$ & 0.16845 & $\tau_{1}$ & 1.21 \\
$\alpha_{2}$ & 0.3181 & $\tau_{2}$ & 19.73 \\
$\alpha_{3}$ & 0.31483 & $\tau_{3}$ & 113.27 \\
$\alpha_{4}$ & 0.17621 & $\tau_{4}$ & 396.38 \\
$\alpha_{5}$ & 0.05149 & $\tau_{5}$ & 1565.40 \\
\hline
\end{tabular}

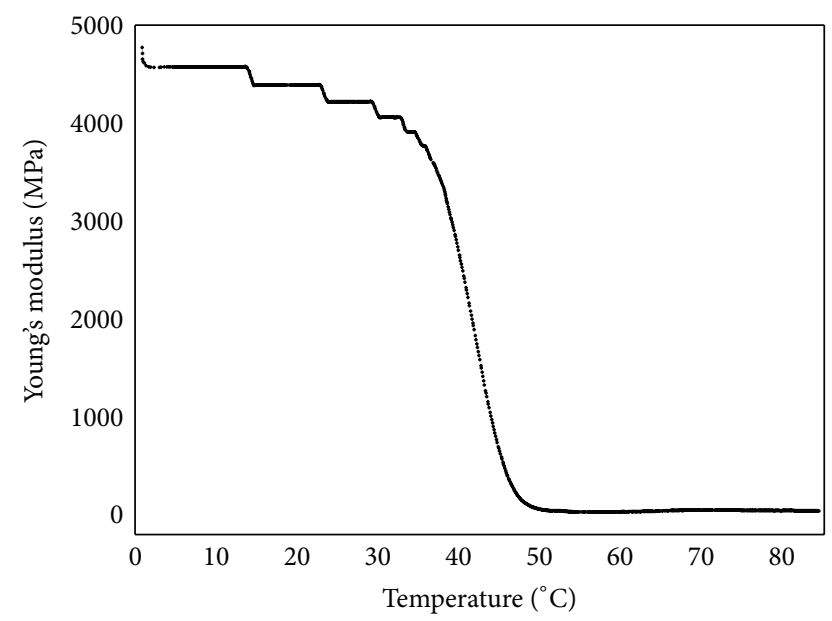

FIGURE 5: Elastic modulus-temperature curve of PLGA.

in Figure 4. The correlation coefficient of the fitting curve and the experimental curve is 0.9987 , which indicates that the generalized Maxwell model can simulate the relaxation behavior of PLGA material accurately. The parameters of generalized Maxwell model with 5 units based on fitting curve are listed in Table 1.

2.4. Elastic Modulus Testing. Elastic modulus of PLGA at different temperatures was tested by the DMA experiments. An advantage of DMA is that we can obtain the modulustemperature curve of the polymer through only one time experiment. PLGA test sample of $32 \times 8.6 \times 3 \mathrm{~mm}$ was fabricated in advance. The DMA experiment was run at $1 \mathrm{~Hz}$, and the temperature was raised from 0 to $85^{\circ} \mathrm{C}$ with the increasing rate of $3^{\circ} \mathrm{C} / \mathrm{min}$. The elastic modulus of DMA result is shown in Figure 5. The glass transition of PLGA can be seen at $40 \sim 45^{\circ} \mathrm{C}$, and then the modulus curve presents a rubbery-melting plateau above $50^{\circ} \mathrm{C}$.

The data from Table 1 and Figure 5 and the coefficients of $C_{1}$ and $C_{2}$ in WLF function provide the basis for the subsequent simulating research on the forming process of PLGA.

\section{Finite Elements Simulations}

3.1. Modeling. The finite element software ABAQUS/STANDARD was used to conduct the simulation. The embossing process was regarded as the quasi-static process.

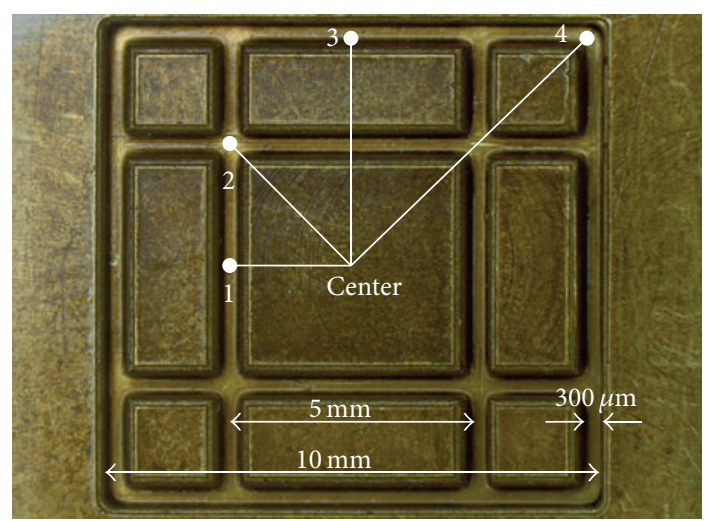

FIGURE 6: The copper mold with square patterns.

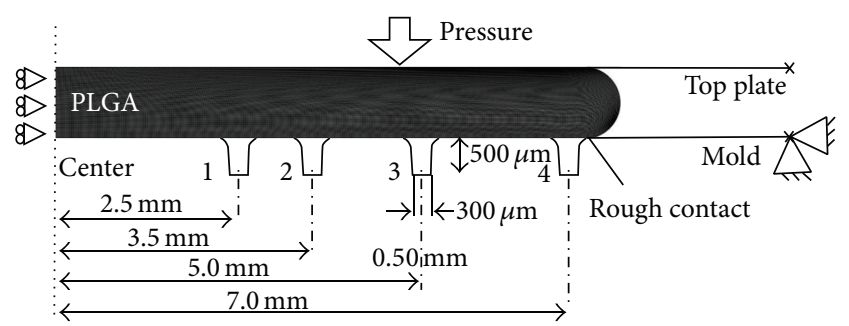

FigURE 7: Simplified plane strain model of the simulation.

Figure 6 shows the copper mold with square patterns. Four different grooves of the patterns on the mold, numbered from 1 to 4 , were chosen according to their distances from the pattern center, to be simplified as the plane strain model, as shown in Figure 7. The top plate and the mold were simplified as rigid bodies, and the fillet edges were modeled to aid in convergence. The interactions between the PLGA materials and the rigid bodies were set as rough contact pairs. The pressure was applied on the top plate, with the mold fixed. The initial thickness of PLGA was set as $1 \mathrm{~mm}$. The PLGA was meshed with linear-order plane strain element CPR4R with the size of 0.02. Dynamic implicit steps were used to analyse the embossing process. Besides, the influence of pressure caused by the trapped air in the grooves was neglected considering its higher computing cost.

3.2. Filling Rules. The pressure of $0.5 \mathrm{MPa}$ was applied on the top plate within $3 \mathrm{~min}$, and the temperature of the PLGA material was set as $90^{\circ} \mathrm{C}$, to simulate the embossing process.

The filling situations at two moments during hot embossing are shown in Figure 8. It can be seen that the nearest groove 1 is filled fully at the time of $22 \mathrm{~s}$, but the uttermost groove 4 does not form fully until $159 \mathrm{~s}$. The colors indicate the pressure levels inside the PLGA, which differs at different places and changes as the embossing moves on. The central pressure keeps the largest and decreases to zero along the radial direction. The pressure-time curves of the nodes above the four grooves are plotted in Figure 9. $x$-axis is the time, and $y$-axis is the pressure value of the chosen node at every moment. It can be seen that the inner groove pressure is kept at a larger value all the time. But the outer groove pressure 


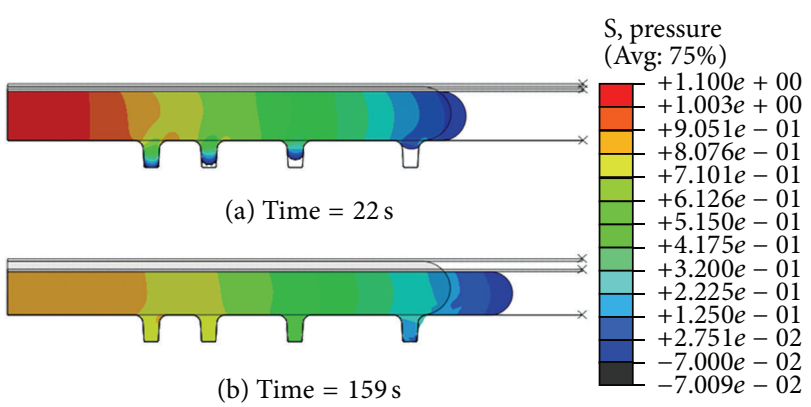

Figure 8: Filling situations at different times.

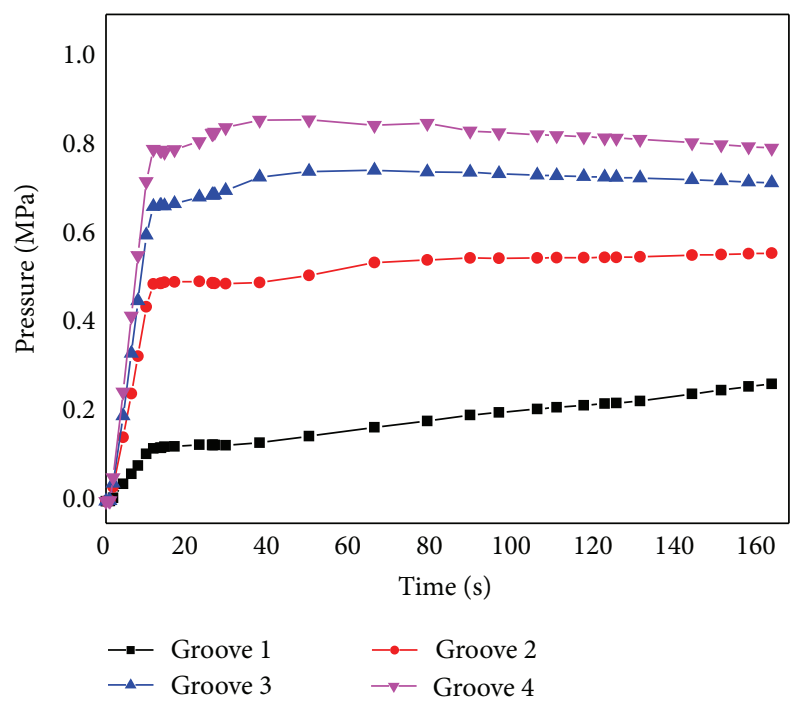

FIGURE 9: Pressure-time curves of nodes above four grooves.

like groove 4, though increasing a little bit later, remains at small values. The filling depth-time curves of the four grooves are plotted in Figure 10. $y$-axis is the depth value of every moment when the PLGA material goes down into the grooves. The slopes of the lines represent the filling speeds at different grooves. The filling speed decreases from groove 1 to 4 , clearly the larger pressure leads to a faster filling speed, and the smaller pressure leads to a slower one.

So the differences of the filling speeds at various grooves are mainly caused by the pressure nonuniformity. Since the filling speed at groove 4 is the smallest one, the duration to fill groove 4 decides how much time is needed to complete the hot embossing. Furthermore, it is necessary to maintain enough molding time to let the microstructures be filled completely. The durations needed to fill groove 4 at various embossing parameters are shown in Figure 11. Apparently, it takes very short time to complete the microstructures at higher temperature, because of the increased fluidity of PLGA. The influence of pressures on the duration domains gradually with the temperature goes down. Higher temperature and pressure can both shorten the fabrication duration. Some of the simulations cannot finish or the computation cannot converge below $80^{\circ} \mathrm{C}$.

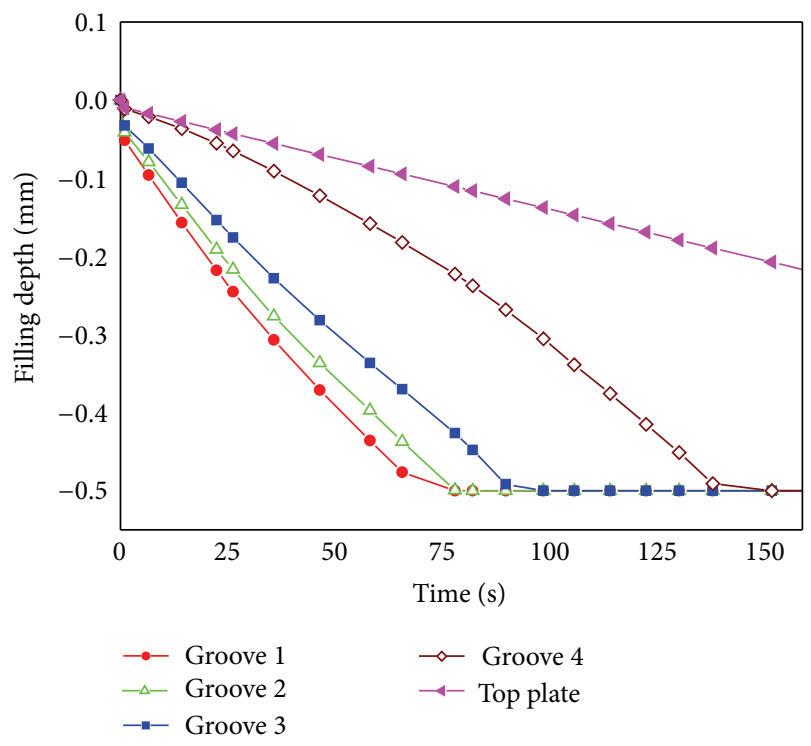

FIgURE 10: Filling depth-time curves of four grooves.

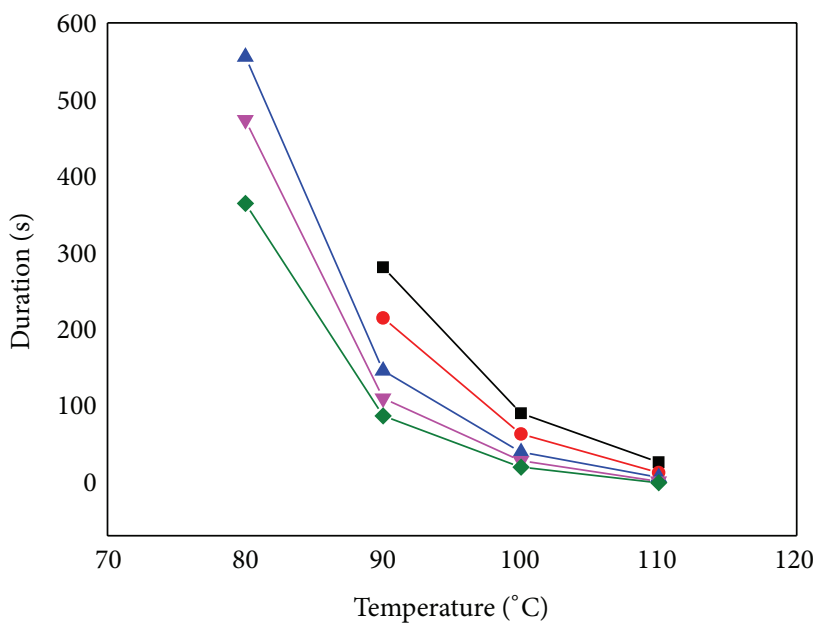

Pressure (MPa)

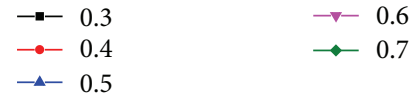

FIGURE 11: Durations to complete the filling.

3.3. Nonisothermal Hot Embossing. Traditionally, the top plate and the mold are both heated to the same temperature during the hot embossing process, which is called the isothermal hot embossing. In this situation, the polymer is heated up and down and is able to reach the defined temperature very quickly. But the process under higher temperature can often lead to material overflow, which decreases the thickness of the embossed microstructures. The overflowing materials then will be cut and wasted.

The nonisothermal hot embossing is proposed to prevent the overflow, which means heating one side of the polymer with the other side being kept cold. So in the experiments, the mold temperature was set as $90^{\circ} \mathrm{C}$ and the top plate 


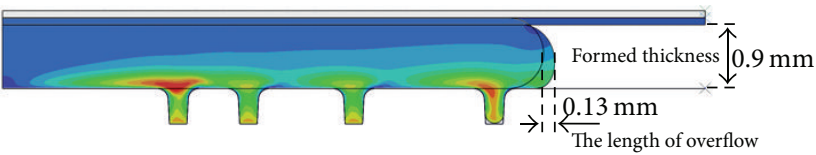

(a) Nonisothermal

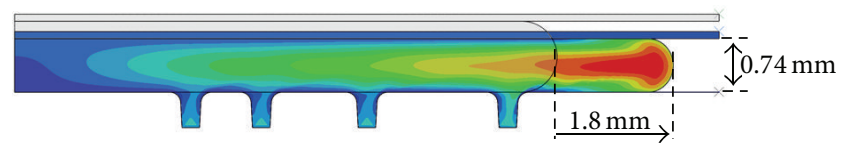

(b) Isothermal

FIGURE 12: Comparison of nonisothermal and isothermal hot embossing.

temperature was set as $20^{\circ} \mathrm{C}$; the temperature inside the PLGA decreased from bottom to top linearly. The other parameters kept the same with the isothermal simulation. The comparison of simulation results is shown in Figure 12. Compared with the isothermal hot embossing, the length of overflow decreased dramatically and the formed thickness increased as well, which proved an effective improvement. The high temperature on the bottom increased the fluidity of PLGA to facilitate the filling, and the low temperature on the top decreased the fluidity of PLGA to prevent overflow. However a longer duration was needed to get the microstructure filled completely during nonisothermal hot embossing.

\section{Experiments and Discussion}

4.1. Effect of Durations. The semifinished PLGA sheets with the size of $10 \times 10 \times 1 \mathrm{~mm}$ were fabricated in advance and then put on the mold to be embossed. Several different hot embossing durations of $1 \mathrm{~min}, 2 \mathrm{~min}$, and $3 \mathrm{~min}$ were applied with the load $50 \mathrm{~N}$ and the temperature $90^{\circ} \mathrm{C}$. After that, cool the equipment to $20^{\circ} \mathrm{C}$, open the plate, and separate the PLGA parts from the mold. The formed microstructures were examined under the microscope (OLYMPUS-SZX16), as shown in Figure 13. The results show that the inner structures can always form quickly, but the outer corners of the parts cannot form fully in short duration. The corner structures were missed in the formed part of 1 min duration, as shown in Figure 13(a). The incomplete microstructure was filled with drugs and sealed with PLGA films and then put into saline as the analog of human body environment. Since the height of the outer structures did not meet the requirement to be sealed, the phenomena of leaks happened at the corners of the carrier, as shown in Figure 13(b). Given that the filling speed at the outer corner was slower than that at the inner one, the longer duration of at least $3 \mathrm{~min}$ was necessary to get complete structures, as shown in Figure 13(c). The experiments agree with simulation results well.

4.2. Defects of Bubbles. The vacuum equipment was not installed in our equipment considering its high cost, so the air would be trapped in the grooves of the mold. Trapped air not only impeded the PLGA materials to fill the grooves, but also developed to the defects of bubbles existed in the microstructures.
A quarter part of the microstructure was observed under the microscope, as shown in Figure 14. The simulation results told us that the pressure of inner places was much higher than the outer places of the mold, so the filling speed of inner grooves was faster than that of the outer grooves during hot embossing. The filling completion would be achieved at the inner structures firstly and then moved on to the outer corners and edges. As a result, the trapped air converged at the cross points of the microstructures and was compressed into visible bubbles. The outer structure was observed under the scanning electron microscope (HITACHI-S-3000N), and the defect of a 3-dimensional bubble cavity can be seen in Figure 15.

A large amount of PLGA microstructures was embossed under various durations from $1 \mathrm{~min}$ to $3 \mathrm{~min}$, with the load of $50 \mathrm{~N}$ and the temperature of $90^{\circ} \mathrm{C}$, then observed, and compared under the microscope. Three rules of the bubbles' formation are drawn as follows.

(1) Microstructure deficiencies were caused by the large bubbles if the pressure and the embossing duration were not sufficient enough, as shown in Figure 16(a).

(2) As the embossing process moved on, the pressure at the corners increased gradually and some air was able to escape to the outside of the grooves, as shown in Figures 16(b) and 16(c).

(3) The rest of the air was compressed into smaller bubbles, which however cost much longer time, as shown in Figures 16(d) and 16(e). Also it is difficult to let the bubbles disappear completely.

4.3. Nonisothermal Hot Embossing Experiment. The temperature at the top plate was set as $20^{\circ} \mathrm{C}$, and the bottom mold was set as $90^{\circ} \mathrm{C}$ when using nonisothermal hot embossing. The load was set as $50 \mathrm{~N}$ and the duration was $3 \mathrm{~min}$. The PLGA microstructures were fabricated by both isothermal and nonisothermal hot embossing methods and compared in Figure 17. It can be seen that the overflow area of the formed part using nonisothermal way is very limited, while the overflow area is much larger when using the isothermal way.

\section{Conclusions}

In this research, the embossing processes of the PLGA microstructures were studied in a combination of simulation and the experimental method. Firstly, the mechanical properties of PLGA were achieved by the material testing experiments. The generalized Maxwell model was built to simulate the viscoelastic behavior during hot embossing. Secondly, the simulation of hot embossing process was conducted using ABAQUS software. Since the pressure inside the material decreased along the radial direction of the pattern, the filling speed differed at different grooves of the mold. The formation of the inner structures of the pattern was always faster than the outer structures. And the higher temperature could increase the fluidity of PLGA and shorten the durations 


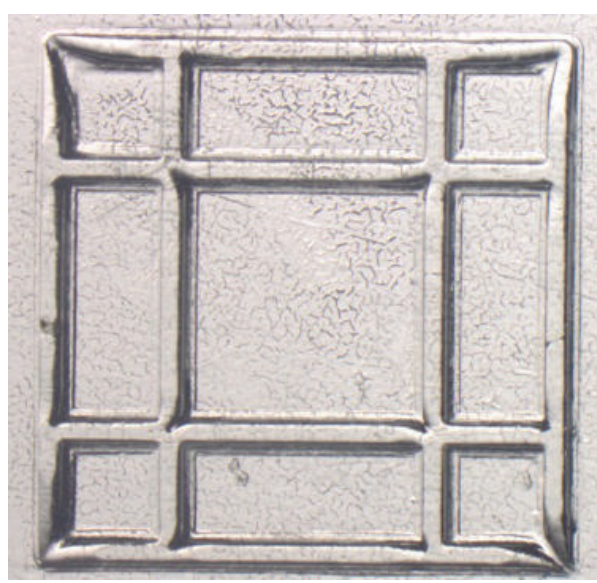

(a)

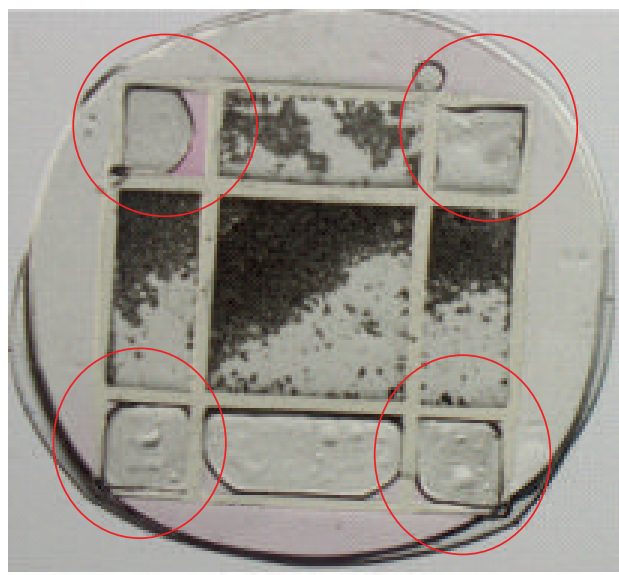

(b)

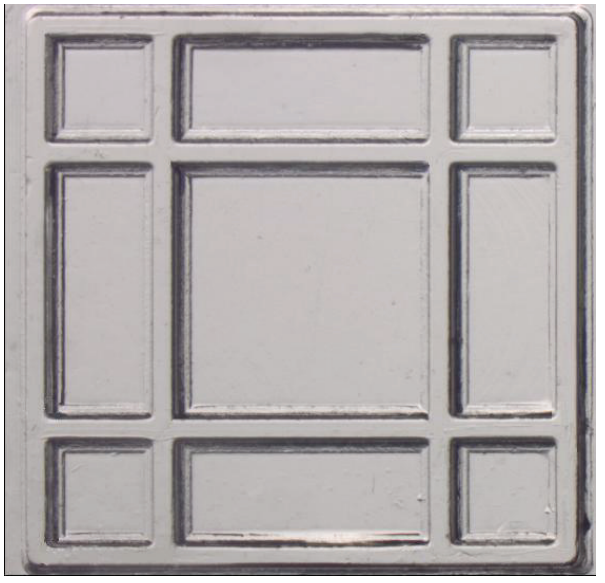

(c)

FIgURE 13: Experimental microstructures under microscope. (a) Short duration of $1 \mathrm{~min}$; (b) leaks at the corners; (c) long duration of 3 min.

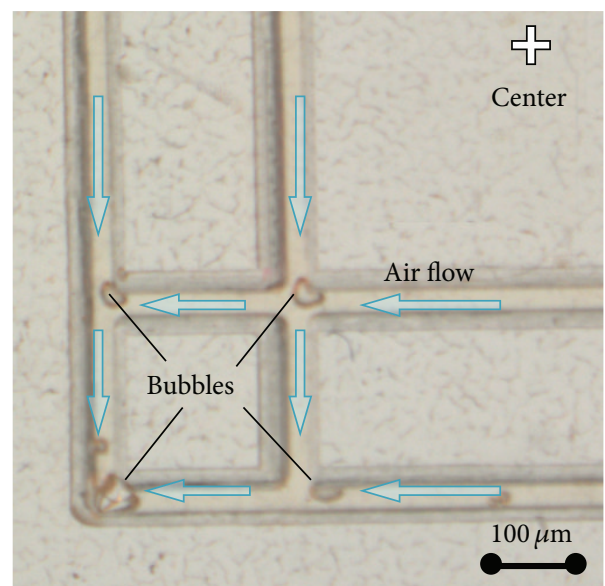

FIgURE 14: Bubbles' formation.

needed. Besides, the nonisothermal hot embossing was proposed to prevent the overflow. Finally, the experiments were presented to verify the simulation results. The incompletion of the structures was caused at the corners because of the lower filling speeds. Moreover, the trapped air was easy to

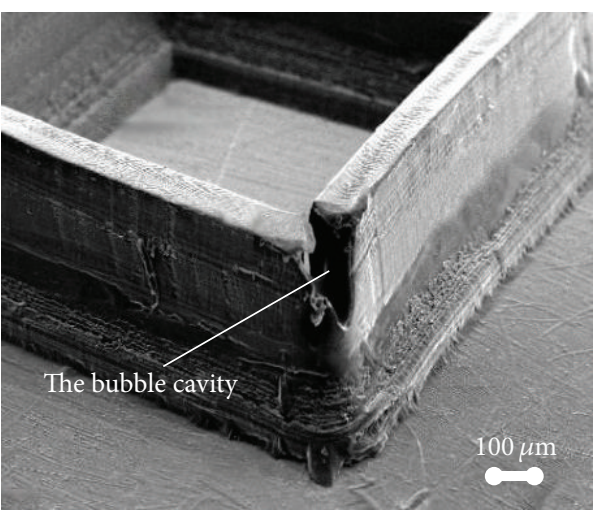

Figure 15: The SEM picture of a bubble defect.

converge at the corners of the mold, which lead to bubble defects. The bubbles could be compressed into smaller ones under longer embossing durations but could not disappear completely. So enough duration was necessary to get the complete and high-quality microstructures. The experiments also showed that the nonisothermal hot embossing impeded the material overflow effectively. 


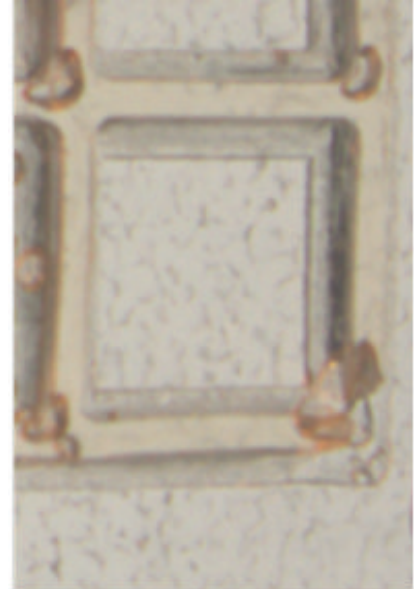

(a)

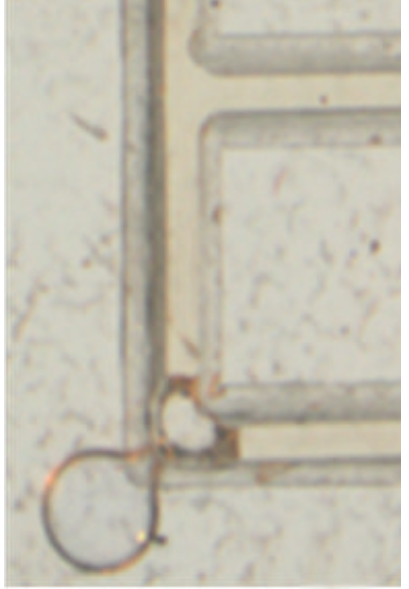

(b)

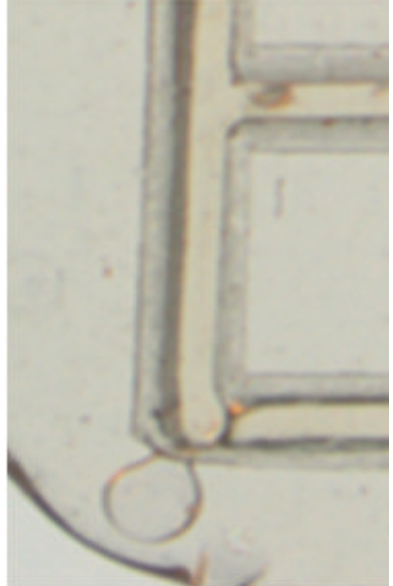

(c)

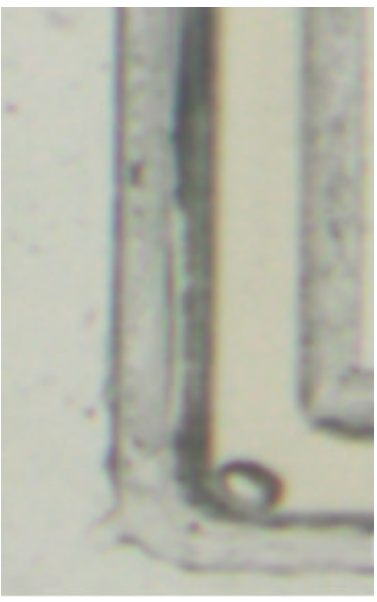

(d)

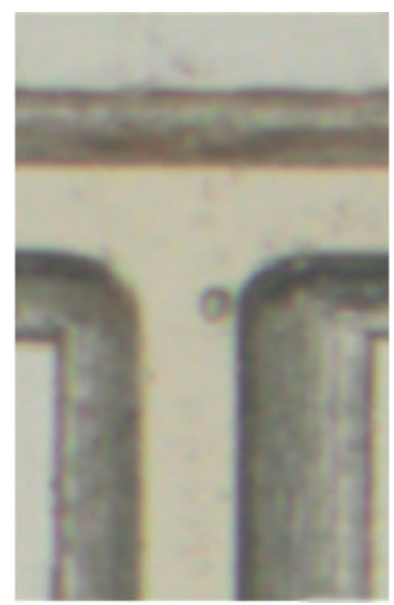

(e)

Figure 16: Bubbles' formation rules (all the edges in the figures have the width of about $300 \mu \mathrm{m}$ ). (a) Microstructure deficiencies; (b), (c) escaping air; (d), (e) compressed small bubbles.

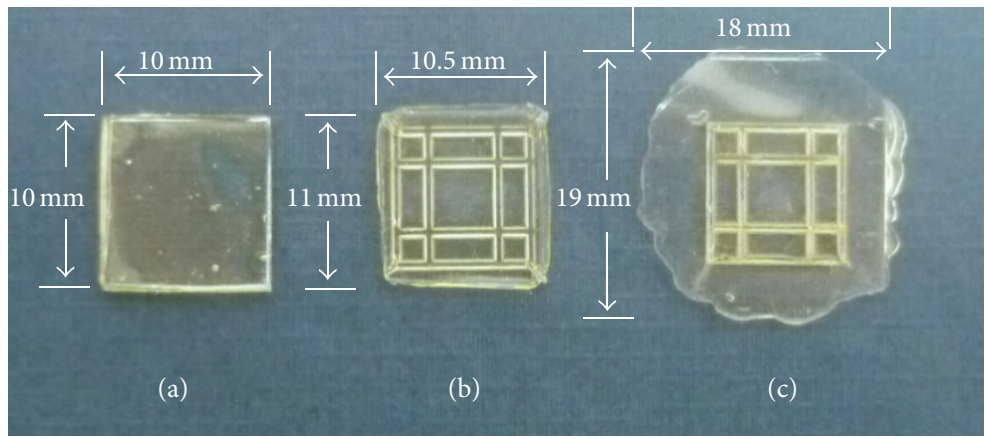

FIGURE 17: Comparison of the overflow areas. (a) Semifinished PLGA sheet; (b) nonisothermal hot embossed microstructure; (c) isothermal hot embossed microstructure.

\section{Conflict of Interests}

The authors declare that there is no conflict of interests regarding the publication of this paper.

\section{Acknowledgments}

This work was supported by National Natural Science Foundation of China (no. 50705074), Collaborative Innovation 
Center of Suzhou Nano Science and Technology, and Fundamental Research Funds for the Central Universities.

\section{References}

[1] M. Heckele and W. K. Schomburg, "Review on micro molding of thermoplastic polymers," Journal of Micromechanics and Microengineering, vol. 14, no. 3, pp. R1-R14, 2004.

[2] M. Worgull, M. Heckele, J. F. Hétu, and K. K. Kabanemi, "Modeling and optimization of the hot embossing process for microand nanocomponent fabrication," Journal of Microlithography, Microfabrication and Microsystems, vol. 5, no. 1, Article ID 011005, 2006.

[3] J. S. Jha and S. S. Joshi, "Numerical simulation of micro hot embossing of polymer substrate," International Journal of Precision Engineering and Manufacturing, vol. 13, no. 12, pp. 2215-2224, 2012.

[4] M. Sahli, T. Barrière, J. C. Gelin, F. Barlat, Y. H. Moon, and M. G. Lee, "Experimental analysis and numerical simulation of the flow behaviour of thin polymer films during hot embossing," AIP Conference Proceedings, vol. 1252, pp. 651-658, 2010.

[5] H. D. Rowland and W. P. King, "Polymer deformation and filling modes during microembossing," Journal of Micromechanics and Microengineering, vol. 14, no. 12, pp. 1625-1632, 2004.

[6] Y. He, J.-Z. Fu, and Z.-C. Chen, "Optimization of control parameters in micro hot embossing," Microsystem Technologies, vol. 14, no. 3, pp. 325-329, 2008.

[7] S. Lan, H.-J. Lee, E. Kim et al., "A parameter study on the micro hot-embossing process of glassy polymer for pattern replication," Microelectronic Engineering, vol. 86, no. 12, pp. 2369-2374, 2009.

[8] L. J. Heyderman, H. Schift, C. David, J. Gobrecht, and T. Schweizer, "Flow behaviour of thin polymer films used for hot embossing lithography," Microelectronic Engineering, vol. 54, no. 3-4, pp. 229-245, 2000.

[9] Y. Hirai, T. Konishi, T. Yoshikawa, and S. Yoshida, "Simulation and experimental study of polymer deformation in nanoimprint lithography," Journal of Vacuum Science and Technology B: Microelectronics and Nanometer Structures, vol. 22, no. 6, pp. 3288-3293, 2004.

[10] H. Taylor, Y. C. Lam, and D. Boning, "An investigation of the detrimental impact of trapped air in thermoplastic microembossing," Journal of Micromechanics and Microengineering, vol. 20, no. 6, Article ID 065014, 2010.

[11] D. Morihara, Y. Nagaoka, H. Hiroshima, and Y. Hirai, "Numerical study on bubble trapping in UV nanoimprint lithography," Journal of Vacuum Science and Technology B: Microelectronics and Nanometer Structures, vol. 27, no. 6, pp. 2866-2868, 2009.

[12] N. Roos, M. Wissen, T. Glinsner, and H.-C. Scheer, "Impact of vacuum environment on the hot embossing process," in Microlithography 2003: International Society for Optics and Photonics, pp. 211-218, 2003.

[13] M. le Berre, J. Shi, C. Crozatier, G. Velve Casquillas, and Y. Chen, "Micro-aspiration assisted lithography," Microelectronic Engineering, vol. 84, no. 5-8, pp. 864-867, 2007.

[14] M. J. Choi, J. Y. Park, K. J. Cha, J.-W. Rhie, D.-W. Cho, and D. S. Kim, "Micropattern array with gradient size (PAGS) plastic surfaces fabricated by PDMS (polydimethylsiloxane) moldbased hot embossing technique for investigation of cell-surface interaction," Biofabrication, vol. 4, no. 4, Article ID 045006, 2012.
[15] Y. J. Juang, L. Lee James, and K. W. Koelling, "Hot embossing in microfabrication. Part I: experimental," Polymer Engineering \& Science, vol. 42, no. 3, pp. 539-550, 2002.

[16] D. Yao, V. L. Virupaksha, and B. Kim, "Study on squeezing flow during nonisothermal embossing of polymer microstructures," Polymer Engineering \& Science, vol. 45, no. 5, pp. 652-660, 2005.

[17] M. Hug, D. Rieser, P. Manns, and G. Kleer, "Investigations on process parameters influencing the quality of optical lenses formed by non-isothermal embossing of inorganic glasses," in Optical Fabrication, Testing, and Metrology II, vol. 5965 of Proceedings of SPIE, Jena, Germany, 2005.

[18] M. Ochoa, C. Mousoulis, and B. Ziaie, "Polymeric microdevices for transdermal and subcutaneous drug delivery," Advanced Drug Delivery Reviews, vol. 64, no. 14, pp. 1603-1616, 2012.

[19] Y. Lu and S. C. Chen, "Micro and nano-fabrication of biodegradable polymers for drug delivery," Advanced Drug Delivery Reviews, vol. 56, no. 11, pp. 1621-1633, 2004.

[20] A. C. Richards Grayson, I. S. Choi, B. M. Tyler et al., "Multipulse drug delivery from a resorbable polymeric microchip device," Nature Materials, vol. 2, no. 11, pp. 767-772, 2003.

[21] X.-P. Wang, T.-N. Chen, and Z.-X. Yang, "Modeling and simulation of drug delivery from a new type of biodegradable polymer micro-device," Sensors and Actuators A: Physical, vol. 133, no. 2, pp. 363-367, 2007.

[22] Y. Gao, X. P. Wang, and T. N. Chen, "Characteristics of biodegradable implantable drug delivery system with microporous structure," in Nems/Mems Technology and Devices, S. Teo, A. Q. Liu, H. Li, and B. Tarik, Eds., pp. 307-310, 2009.

[23] S. Lan, H.-J. Lee, S.-H. Lee et al., "Experimental and numerical study on the viscoelastic property of polycarbonate near glass transition temperature for micro thermal imprint process," Materials and Design, vol. 30, no. 9, pp. 3879-3884, 2009.

[24] M. Patel, M. Soames, A. R. Skinner, and T. S. Stephens, "Stress relaxation and thermogravimetric studies on room temperature vulcanised polysiloxane rubbers," Polymer Degradation and Stability, vol. 83, no. 1, pp. 111-116, 2004. 

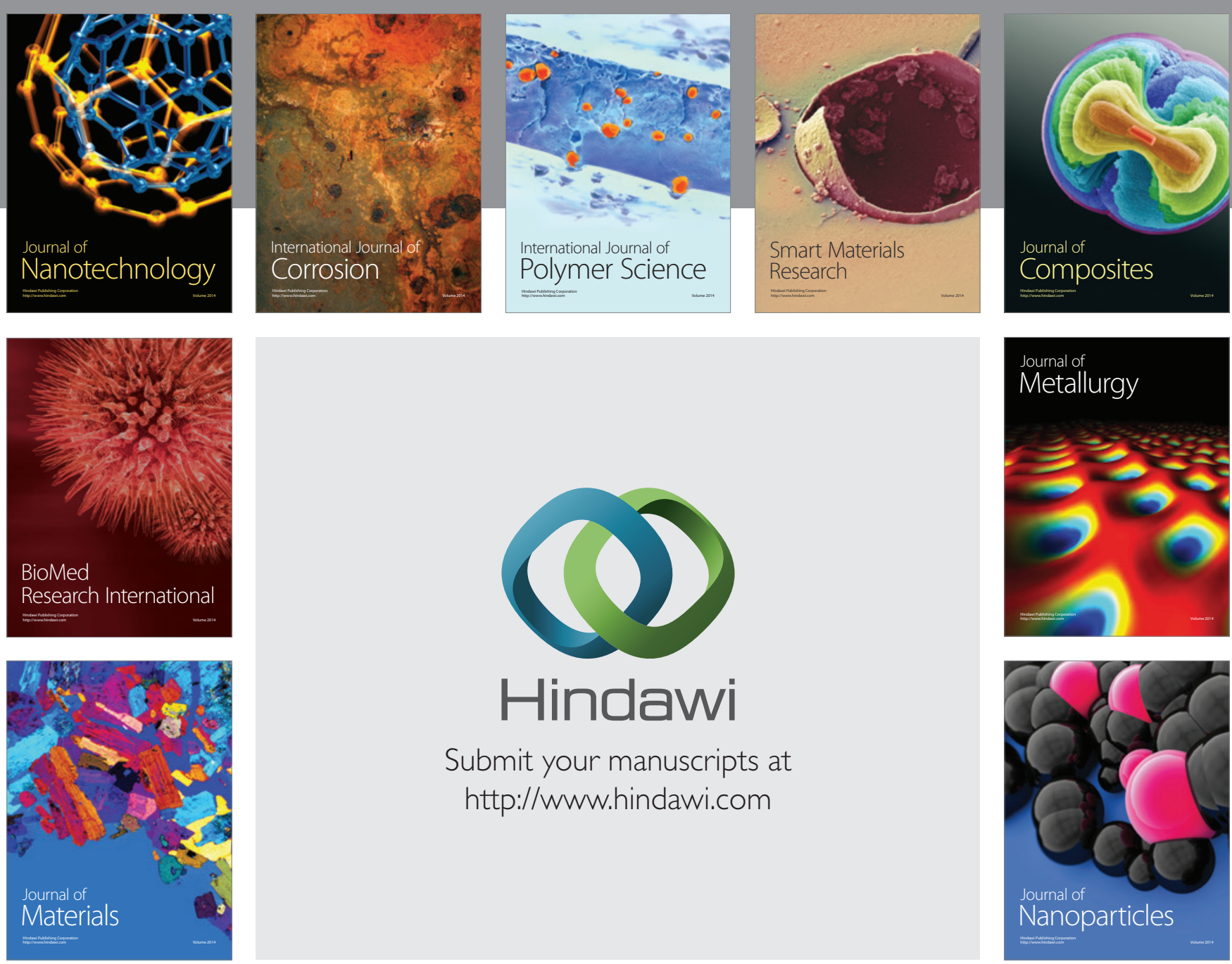

Submit your manuscripts at http://www.hindawi.com
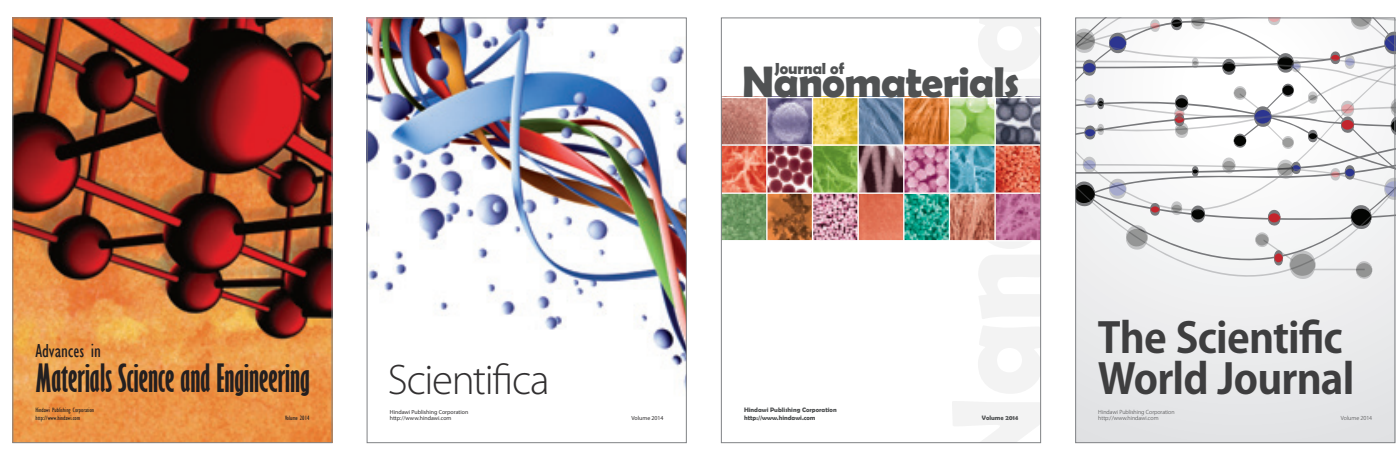

\section{The Scientific World Journal}
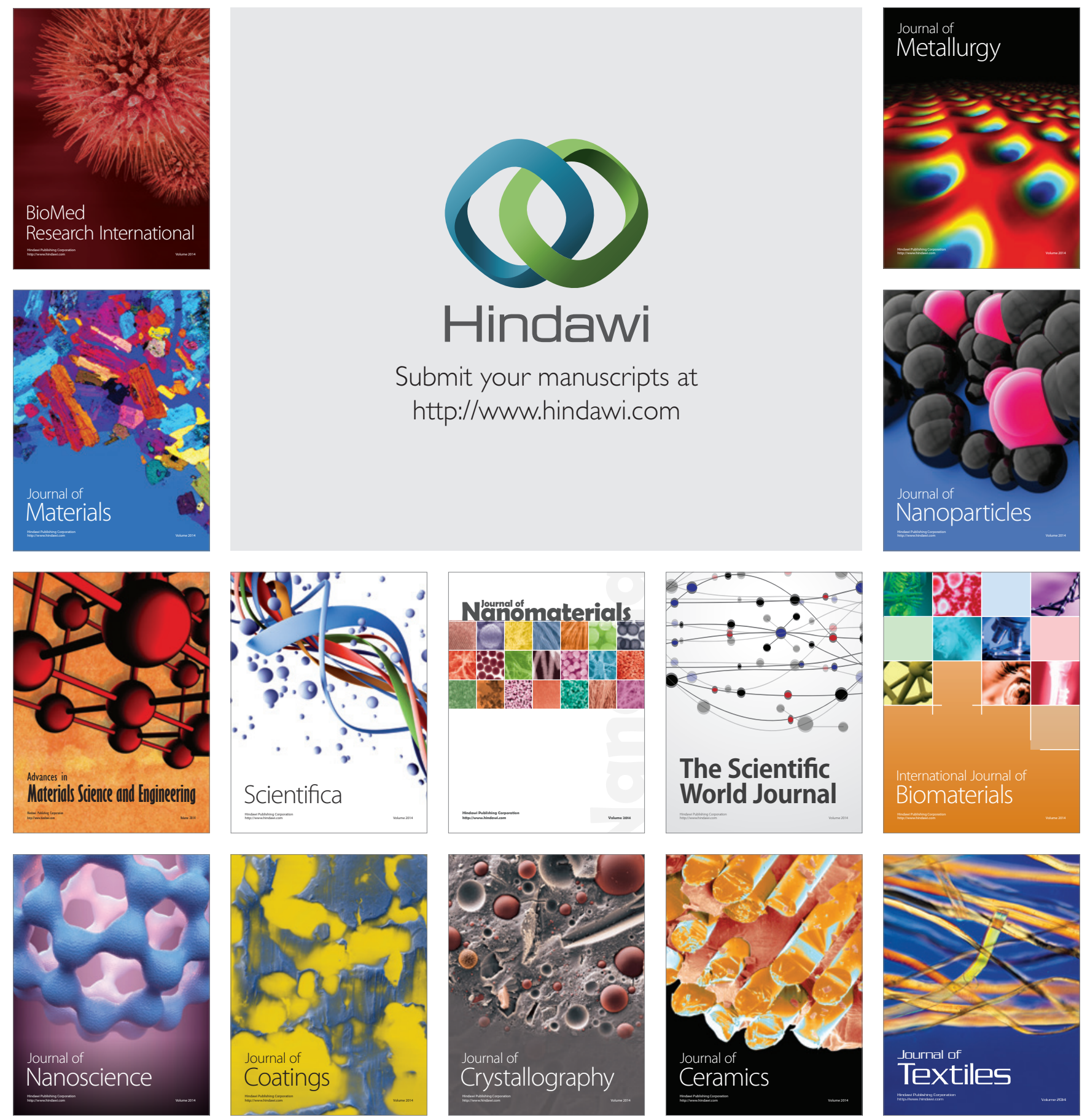